\title{
LE TRANSPORT MARITIME DEPUIS 1945 : FACTEUR CLÉ DE LA MONDIALISATION
}

\author{
par Antoine FRÉMONT \\ Directeur de recherche en géographie \\ Directeur scientifique adjoint \\ Institut français des sciences et technologies des transports, \\ de l'aménagement et des réseaux (IFSTTAR)
}

\begin{abstract}
Aux fantasmes d'un monde virtuel né de l'Internet s'oppose la réalité d'une mondialisation synonyme de flux massifs de marchandises d'une ampleur inégalée depuis la fin de la Seconde Guerre mondiale. Le transport maritime, tout particulièrement la conteneurisation, a été l'un des facteurs clés de cette mondialisation en accompagnant la croissance des échanges internationaux et en contribuant à redessiner l'espace productif mondial. Mondialisée, cette industrie maritime pose des questions de gouvernance mondiale.
\end{abstract}

« La mondialisation, c'est l'échange généralisé entre les différentes parties de la planète, l'espace mondial étant alors l'espace de transaction de l'humanité. Simultanément, croissances et changements accompagnent ce développement des échanges globaux. Jamais dans son histoire, l'humanité n'a connu une accélération de changements aussi forte qu'au cours du dernier siècle et surtout des cinquante dernières années. » Ainsi est décrit le processus de mondialisation par le géographe Olivier Dollfus en 1997 et son accélération depuis la fin de la Seconde Guerre mondiale ${ }^{1}$.

Le transport maritime joue un rôle clé dans cette interconnexion généralisée du monde : plus de $80 \%$ du commerce international en volume est acheminé par la voie maritime, ce qui représente aujourd'hui plus de 10 milliards de tonnes transportées par la voie maritime contre 550 millions seulement en 1950. À l'intérieur du transport maritime, la conteneurisation élargit aux produits manufacturés et semi-finis cette capacité presque infinie de transport fiable à très bas coût sur de très longues distances.

Mais le constat ne se limite pas simplement à l'accompagnement du développement des échanges par le transport maritime. Ce dernier contribue aussi à remodeler l'espace productif mondial. Le déploiement à l'échelle internationale des chaînes de la valeur est rendu physiquement possible : concevoir un produit dans un centre de R\&D d'un pays riche, produire ses composants là où les compétences et le savoirfaire industriel existent, l'assembler dans un pays où le coût de la main-d'œuvre est faible, le distribuer dans les pays où les consommateurs disposent d'un pouvoir d'achat. Pour y parvenir, l'industrie du transport maritime en tant que secteur d'activité économique, avec ses règles spécifiques de fonctionnement et ses propres acteurs, se transforme profondément.

${ }^{1}$ O. Dollfus, La mondialisation, Paris, Presses de Sciences Po, 1997. 
Les réseaux maritimes se mondialisent. Pour résumer l'hypothèse simplement, sans transport maritime et sans conteneurisation, pas de mondialisation.

Nous montrerons dans un premier temps comment le transport maritime a gagné en efficacité et productivité depuis la fin de la Seconde Guerre mondiale, ce qui permet un accroissement des échanges globaux. Puis la transformation de l'espace productif mondial par le transport maritime sera mise en évidence sans oublier de souligner les limites, peut-être même les impasses, auxquelles est désormais confronté ce système maritime mondial après des décennies de croissance ininterrompues.

\section{PRODUCTIVITÉ MARITIME ET CROISSANCE DES ÉCHANGES MONDIAUX}

\subsection{Spécialisation des navires et productivité maritime}

À partir de la seconde moitié du XIX ${ }^{\mathrm{e}}$ siècle et tout au long du $X^{\mathrm{e}}$ siècle, la tendance lourde est, avec la fin de la marine à voile, à la spécialisation progressive des navires en fonction des marchandises à transporter. Elle correspond à l'avènement de la marine marchande contemporaine qui permet des acheminements intercontinentaux de plus en plus réguliers, importants et fiables indépendamment des conditions océaniques.

À chaque période son navire de prédilection. Pendant la première moitié du $\mathrm{XX}^{\mathrm{e}}$ siècle et jusqu'à l'avènement de l'aviation commerciale, les paquebots dominent. Ils permettent des relations régulières avec le Nouveau Monde mais aussi avec les empires coloniaux. Après la Seconde Guerre mondiale survient l'avènement des pétroliers et des vraquiers puis des porte-conteneurs. Les vracs liquides, essentiellement le pétrole, sont acheminés grâce à des navires citernes, les vracs secs (minerais, charbon, grains...) grâce à des navires vraquiers alors que les marchandises diverses, celles qui ne sont pas en vrac, sont aujourd'hui largement conteneurisées. Pour les vracs, la règle est celle du tramping. Les navires effectuent des voyages à la demande en fonction des cargaisons à transporter. Leurs itinéraires ne sont pas réguliers et varient d'un voyage à l'autre. Pour les marchandises diverses prévaut la ligne régulière. Les navires suivent des itinéraires précis et cadencés.

\subsection{La révolution du conteneur}

La conteneurisation élargit aux marchandises les plus diverses le principe de la standardisation du navire. L'idée simple et américaine s'inscrit dans la droite ligne du fordisme. Malcolm McLean, transporteur routier à l'origine, la personnifie, dans les années cinquante. Elle a déjà été expérimentée par les militaires américains pendant la Seconde Guerre mondiale avec le transport de marchandises dans des boîtes ou « containers ». Mais McLean l'industrialise : il utilise des boites standardisées de forte capacité qui correspondent au gabarit des remorques routières, longues de 35 pieds. Elles peuvent être empilées (gerbées) sur sept hauteurs, ce qui permet leur stockage vertical dans des cales de navires ou en pontée. Et surtout leur manutention grâce à des grues qui deviendront des portiques spécialisés est aisée grâce aux quatre pièces de coin (twist locks) destinées à saisir le conteneur via un palonnier (spreader).

En avril 1956, l'Ideal X, un navire pétrolier T2 de la Seconde Guerre mondiale transformé par McLean pour transporter ses boîtes, effectue un premier voyage entre le port de Newark et Houston avec 58 boîtes à bord. Alors que les piers de Manhattan grouillent d'activité et reçoivent encore pour quelques années les grands paquebots transatlantiques, le lieu de l'innovation se déroule sur la rive opposée de la baie de l'Hudson qui deviendra en quelques décennies le nouveau cœur du port de New York avec l'implantation des terminaux à conteneurs. 
McLean révolutionne la manutention portuaire. Ce ne sont plus les marchandises diverses qui sont manutentionnées mais les boîtes standardisées. Les gains de productivité sont considérables. Les cargos classiques passaient environ $60 \%$ de leur temps dans les ports et pendant les $4 / 5$ de leur séjour dans le port aucune opération de manutention de la cargaison ne se déroulait. Dans un article du 21 janvier 1965, le Financial Times indique que la Matson Line a pu décharger en 850 hommes-heures un navire de 6500 tonnes spécialement aménagé au lieu des 11000 hommes-heures nécessaires pour un navire de type classique et de même tonnage. Les heures de dockers ont été réduites de $90 \%$, la durée de séjour dans le port de $80 \%{ }^{2}$.

Dans les ports, la création de terminaux spécialisés dotés de portiques à conteneurs dédiés à la manutention des boîtes va démultiplier ces gains de productivité portuaires qui sont à l'origine du succès sans précédent de la conteneurisation. L'escale d'un porte-conteneurs ne dure pas au-delà d'une trentaine d'heures. L'armateur qui l'exploite utilise plus rationnellement un outil, le navire, aux coûts fixes très importants.

Les dix premières années de la conteneurisation correspondent à une phase d'apprentissage. De 1956 à 1966, les services maritimes conteneurisés sont limités à des trafics côtiers en Amérique du Nord et entre les États-Unis et l'Alaska, Hawaï et Porto Rico. En 1965, les normes ISO pour les boîtes sont adoptées. L'EVP (Équivalent Vingt Pieds) permet de mesurer la capacité de transport des porte-conteneurs ${ }^{3}$. En 1966 débutent les premiers services transocéaniques et la généralisation progressive de la conteneurisation au monde.

\subsection{Des navires toujours plus grands}

La spécialisation des navires permet d'accroître leur taille afin de bénéficier des économies d'échelle et de réduire le coût à l'unité transportée, que ce soit une tonne de pétrole, de charbon ou d'ordinateurs à l'intérieur d'un conteneur. Les navires se distinguent par leur remarquable capacité à produire de la tonne-kilomètre à très bas coût ${ }^{4}$.

Pendant les Trente Glorieuses, l'augmentation des quantités de pétrole à transporter, la longueur des routes entre le Golfe persique et le Japon ainsi qu'entre le Golfe persique et l'Europe, notamment à la suite de la fermeture du canal de Suez de 1967 à 1975 qui contraint à un contournement de l'Afrique par le cap de Bonne Espérance, expliquent la croissance continue de la capacité de transport des pétroliers. À la veille du premier choc pétrolier de 1973, les grands groupes pétroliers décident la construction des super tankers de 500000 $\mathrm{Tpl}^{5}$ et des navires de $1 \mathrm{M} \mathrm{Tpl}$ sont même sérieusement envisagés. Ils seront démolis au cours de la même décennie faute de cargaisons suffisantes à transporter. Les navires vraquiers suivent la même évolution, les plus gros ayant actuellement un port en lourd ${ }^{6} \mathrm{de}$ 350000 tonnes. La taille des navires utilisés

\footnotetext{
${ }^{2}$ A. Frémont, La French Line face à la mondialisation de l'espace maritime, Paris, Anthropos, collection Géographie, 1998.

${ }^{3}$ E.V.P. ou Équivalent Vingt Pieds (TEU en anglais : Twenty Equivalent Unit) : correspond à un conteneur équivalent vingt pieds. Il existe principalement des conteneurs 20 pieds et 40 pieds. 1 conteneur 40 pieds vaut 2 E.V.P. La capacité de transport des navires porte-conteneurs est donnée en EVP, par exemple un porte-conteneurs de 5000 EVP est capable de transporter 5000 conteneurs équivalents 20 pieds.

${ }^{4}$ K. Cullinane, M. Khanna, "Economies of scale in large containerships: optimal size and geographical implications", Journal of Transport Geography, vol. 8, n 3, 2000, p. 181-195.

${ }^{5} \mathrm{La}$ tonne de port en lourd (Tpl), (Deadweight tonnage en anglais) permet de mesurer la capacité de chargement en poids d'un navire. Celle-ci correspond au nombre de tonnes qu'un navire peut transporter y compris les approvisionnements et les soutes.

${ }^{6} \mathrm{La}$ tonne de port en lourd (Tpl) (Deadweight tonnage DWT) : nombre de tonnes qu'un navire peut transporter y compris les approvisionnements et les soutes qu'il faut enlever si on veut connaître la charge utile.
} 
Tableau 1 : Évolution de la taille des pétroliers

\begin{tabular}{|l|c|c|c|c|c|c|}
\cline { 2 - 7 } \multicolumn{1}{c|}{} & $\mathbf{1 9 4 4}$ & $\mathbf{1 9 5 5}$ & $\mathbf{1 9 6 5}$ & $\mathbf{1 9 6 8}$ & $\mathbf{1 9 7 6}$ & $\begin{array}{c}\text { Paquebot } \\
\text { France }\end{array}$ \\
\hline Tonnes de port en lourd & 17000 & 35000 & 76000 & 127000 & 553662 & 13960 \\
\hline Longueur (mètres) & 160 & 204 & 244 & 274 & 414 & 315 \\
\hline Largeur (mètres) & 21 & 26 & 37 & 42 & 63 & 34 \\
\hline Tirant d'eau (mètres) & 9 & 11 & 13 & 15 & 29 & 10 \\
\hline
\end{tabular}

Source : À partir des rapports annuels d'Armateurs de France divers

varie en fonction de la longueur des routes et des volumes.

Navires totalement standardisés, les porte-conteneurs peuvent techniquement suivre la même évolution ${ }^{7}$. En 40 ans, leur capacité de transport a été multipliée par plus de 20, de 1000 EVP à la fin des années 1960 à plus de 20000 aujourd'hui. Deux grandes étapes peuvent être distinguées dans la croissance de la taille des porte-conteneurs. Jusqu'au milieu des années 1980, leur capacité croît jusqu'à atteindre les limites du gabarit Panamax qui correspond à la largeur des écluses du canal de Panama, soit une capacité maximum de 4500 EVP.

En 1988, l'armement singapourien APL met en service les premiers porte-conteneurs post-panamax dont la largeur excède celle des écluses du canal de Panama ${ }^{8}$. Les armateurs s'affranchissent de la norme Panamax car le canal n'est plus perçu comme un point de passage indispensable des routes maritimes alors que l'Asie orientale, en relation privilégiée avec l'Amérique du Nord et l'Europe, s'affirme comme le nouveau poumon du commerce international.

Deux sauts successifs repoussent encore les limites sous l'impulsion du groupe danois Møller à travers sa filiale Maersk Line, premier armement mondial de lignes régulières conteneurisées. En 2006, l'Emma Maersk (E Class) est le premier porte-conteneurs de plus de 10000 EVP avec une capacité de 14500
EVP. En 2011, Maersk prend commande des Triple E d'une capacité légèrement supérieure à $18000 \mathrm{EVP}$, ouvrant ainsi la voie aux navires actuels de plus de 20000 EVP.

L'accroissement de la capacité des porte-conteneurs se traduit par une augmentation de leur largeur, longueur, tirant d'eau et port en lourd. Par la longueur et la largeur, ils se rapprochent des plus gros navires pétroliers construits dans les années 1970 sans pour autant atteindre leur tirant d'eau et leur port en lourd car ils transportent des marchandises plus légères que le pétrole.

Tout au long de ces décennies, la capacité moyenne de la flotte mondiale conteneurisée ne cesse d'augmenter, tirée par les commandes de navires toujours plus grands.

\subsection{Croissance du commerce international et des échanges par la voie maritime}

Le transport maritime accompagne la croissance du commerce international depuis 1945. Celle-ci s'expliquent par de nombreux facteurs : volonté politique des États-Unis, négociations du GATT en rounds successifs pour abaisser les droits de douane, création en 1995 de l'OMC qui offre un cadre multilatéral de régulation du commerce international, développement des zones régionales de libre échange, en premier lieu l'Union européenne,

\footnotetext{
${ }^{7}$ M. R. Brooks, Sea Change in liner shipping: Regulation and Managerial Decision-Making in a Global Industry, Oxford, Pergamon, 2000.

${ }^{8}$ J. Charlier, « De la norme panamax à l'essor des over panamax », Acta Geographica, n 121, 2000, p. 102-111.
} 
Tableau 2 : Évolution de la taille des porte-conteneurs

\begin{tabular}{|l|c|c|c|c|c|c|}
\cline { 2 - 7 } \multicolumn{1}{c|}{} & $\mathbf{1 9 6 8}$ & $\mathbf{1 9 8 5}$ & $\mathbf{1 9 8 8}$ & $\mathbf{2 0 0 6}$ & $\mathbf{2 0 1 3}$ & $\mathbf{2 0 1 9}$ \\
\cline { 2 - 7 } \multicolumn{1}{c|}{} & \multicolumn{2}{c|}{ Panamax } & \multicolumn{4}{c|}{ Post-Panamax } \\
\hline Capacité (EVP) & 900 & 4458 & 4340 & 15000 & 18000 & 22000 \\
\hline Longueur (m) & 180 & 290 & 275 & 397 & 400 & 400 \\
\hline Largeur (m) & 24 & 32,2 & 39,1 & 56 & 59 & 61,4 \\
\hline Tirant d'eau (m) & 9,1 & 10,7 & 12,5 & 16 & 16 & 16 \\
\hline
\end{tabular}

Sources : Données armateurs divers

Graphique 1 : Évolution de la capacité moyenne des porte-conteneurs en service, mis en service et du plus gros porte-conteneurs depuis 1992 (en EVP)

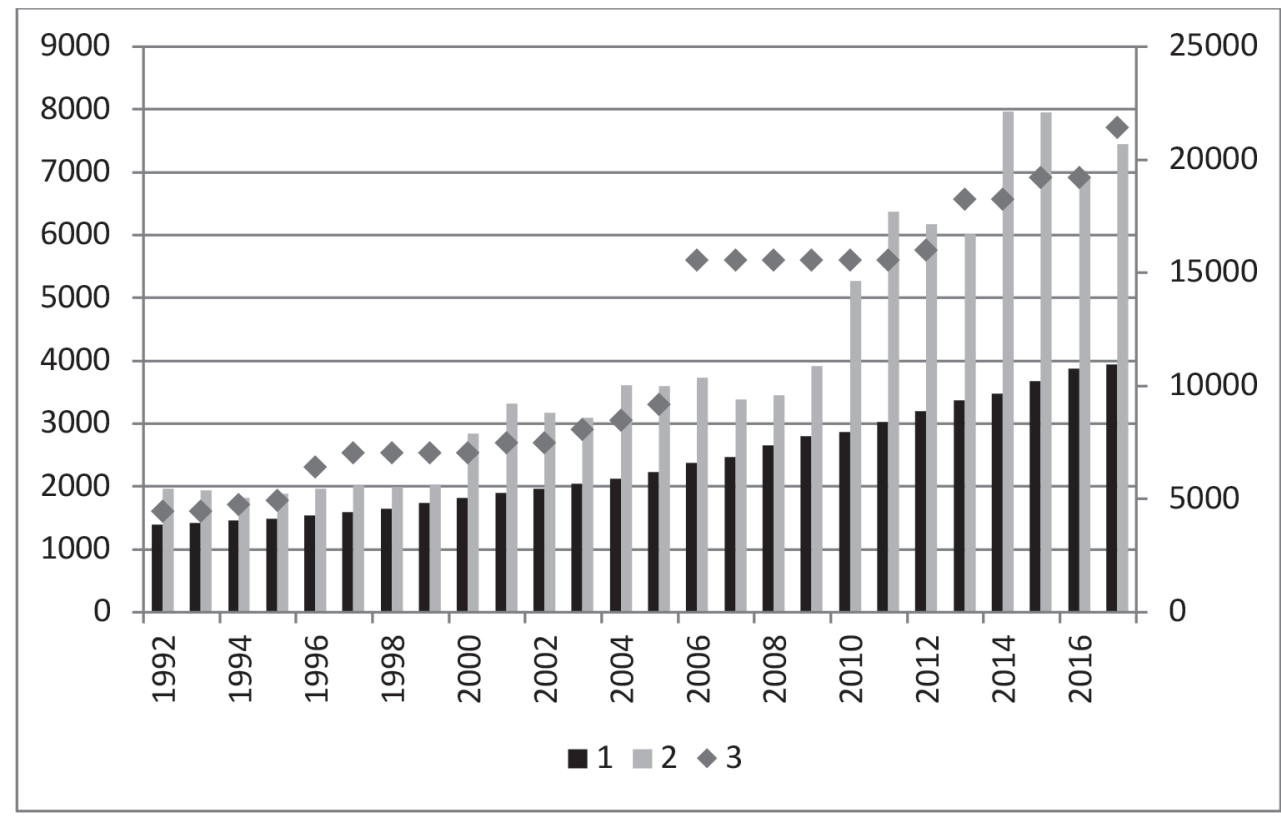

1. Capacité moyenne des porte-conteneurs en service

2. Capacité moyenne des porte-conteneurs mis en service

3. Capacité du plus gros porte-conteneurs (en ordonnée à droite)

Source : Drewry, Container. Forecaster \& Annual Review 2017/18, London, 2018.

essor des firmes multinationales et globalisation des marchés financiers 9 .

En 2016, le commerce international est 36 fois supérieur en volume à ce qu'il était en 1950. Depuis 1945, il croît à une vitesse plus rapide que la production mondiale, ce qui traduit l'interdépendance accrue des économies les unes par rapport aux autres. Ce phénomène s'accélère encore à partir des années 1990 jusqu'à la crise sans précédent

${ }^{9}$ E. Combe, « Le multilatéralisme, du GATT à l'OMC », in Le commerce mondial, Les Cahiers français, $\mathrm{n}^{\circ} 299$, 2000, p. 9-13. 
de 2008 qui marque l'entrée dans une ère plus incertaine. Néanmoins de 1990 à 2016, le commerce international est multiplié par 3,3 en volume malgré la crise de 2008 contre seulement 2,4 de 1970 à 1990 . La Chine après les nouveaux pays industriels d'Asie (NPIA) tire la croissance du commerce international.

Les volumes transportés par la voie maritime ne cessent d'augmenter. Les fluctuations du volume des marchandises transportées par la voie maritime et de la flotte mondiale sont un exact miroir de celles du commerce international. Quelques crises marquantes se lisent parfaitement dans les courbes : chocs pétroliers de 1973 et 1979 suivis du contre choc pétrolier, récession de 2008 sans précédent depuis 1929. L'évolution de la flotte mondiale lisse les tendances car l'adaptation de l'offre de navires à la demande prend du temps, ce qui entraîne des crises récurrentes de surcapacités dans le transport maritime qui se traduisent elles-mêmes par d'importantes fluctuations des taux de fret.

À partir des années 1970, les autres marchandises progressent plus rapidement que les vracs pour devenir à partir des années 1990 le premier type de marchandise transporté par la voie maritime. Aux Trente Glorieuses des industries lourdes succèdent une économie où les produits manufacturés, finis ou semi-finis, sont au cœur des chaînes de valeur. La flotte des porte-conteneurs est au service de cette industrie manufacturière mondialisée. Depuis les années 1980, sa croissance est plus rapide que celle de la flotte mondiale. Sa part dans la flotte mondiale en Tjb s'élève à $19 \%$ aujourd'hui contre 3\% en 1980.

Pour autant pétrole et vracs repartent à la hausse à partir du milieu des années 1980 , tirés notamment par l'appétit croissant de l'économie chinoise en énergie et pondéreux ${ }^{10}$. L'économie mondialisée, dite post-fordiste, n'a jamais été autant matérielle.

Graphique 2 : Évolution du commerce mondial (volume), des marchandises transportées par voie maritime (tonnes) et de la flotte mondiale (Tjb). 1950-2016. 1990= indice 100

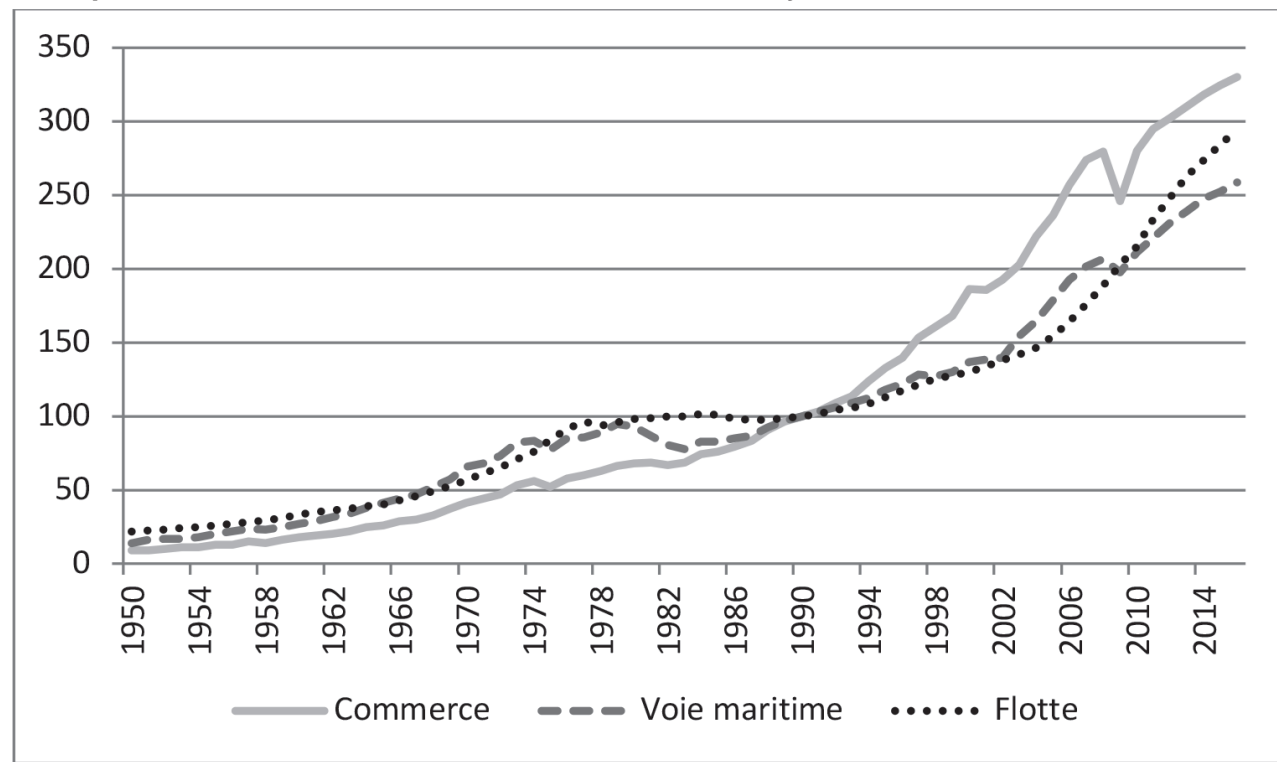

Source : Organisation Mondiale du Commerce (OMC), Institute of Shipping and Logistics

\footnotetext{
${ }^{10}$ United Nations Conference on Trade and Development, Review of Maritime Transport, Geneva, 2018.
} 
Graphique 3 : Transport par la voie maritime depuis 1960 par type de marchandises et en milliards de tonnes

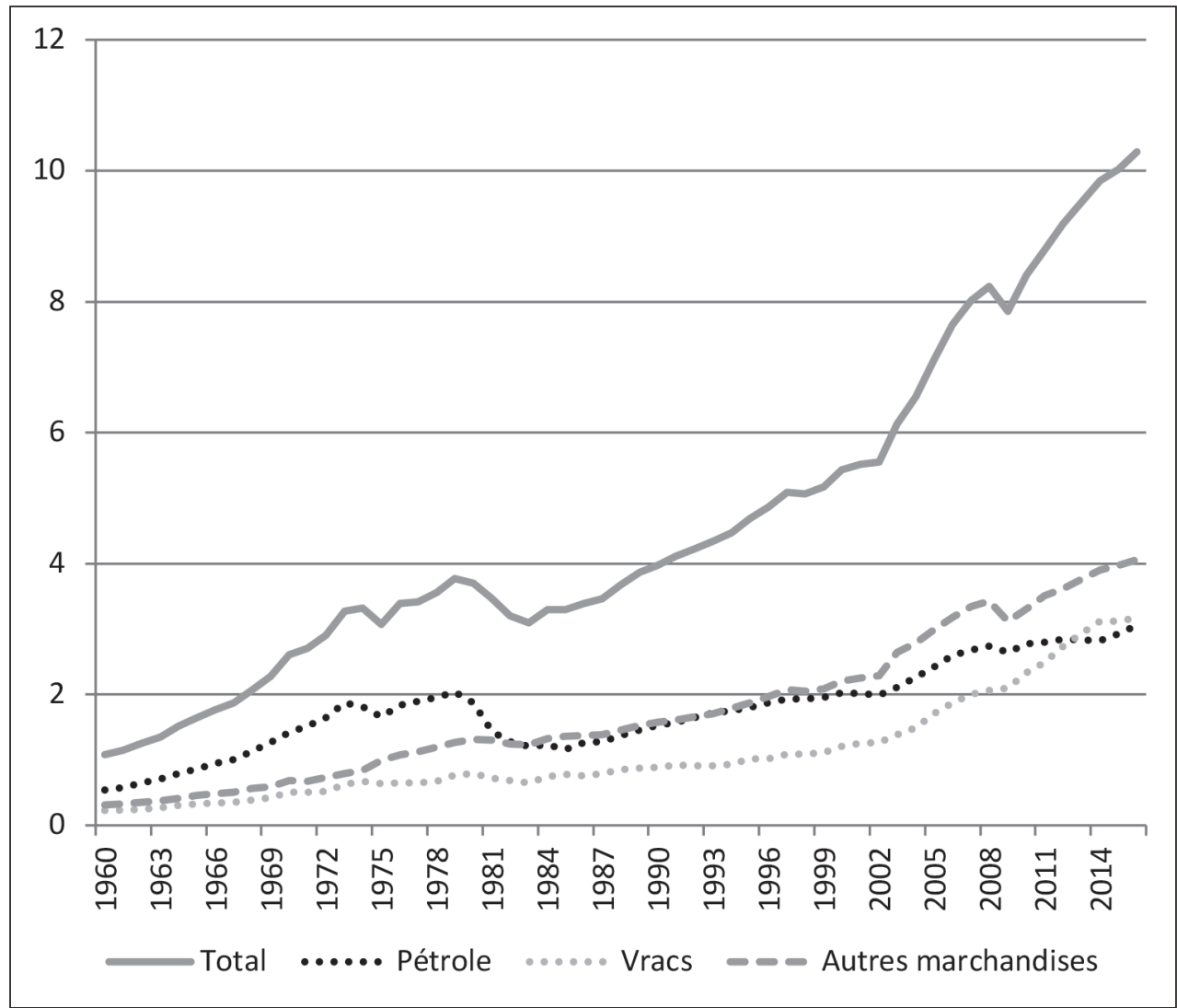

Source : ISL

\section{LATRANSFORMATION DE L'ESPACE PRODUCTIF MONDIAL PAR LA MER}

\subsection{Les Zones Industrialo- Portuaires : logiques nationales puis interdépendance mondiale}

Les zones industrialo-portuaires (ZIP), initiées pendant les Trente Glorieuses et dont le succès ne s'est pas démenti depuis, ont puissamment contribué à la transformation des espaces industriels à différentes échelles, nationalement puis internationalement. Elles regroupent sur des milliers d'hectares des industries lourdes, principalement des complexes pétrochimiques et sidérurgiques. Elles s'appuient sur le principe de l'industrie sur l'eau. Les matières premières arrivent par navires pétroliers ou vraquiers qui accostent littéralement au pied des usines afin de les transformer sur place. Leur transport terrestre trop coûteux est évité. Pour les ports qui en bénéficient, les ZIP créent une rente de trafics car ces derniers sont adossés à l'activité industrielle. Elles nécessitent de vastes espaces 
industriels et portuaires de plusieurs milliers d'hectares ${ }^{11}$.

Deux grandes périodes peuvent être distinguées. La première correspond aux Trente Glorieuses. Les ZIP sont alors l'apanage exclusif des pays riches des deux premières révolutions industrielles en Europe, en Amérique du Nord et au Japon. Elles répondent en Europe et au Japon à une volonté planificatrice de puissance et d'indépendance nationale dans le contexte de la reconstruction puis de haute croissance. Elles s'appuient préférentiellement sur des groupes industriels nationaux. Pour la sidérurgie comme pour le raffinage et la pétrochimie, les ZIP aboutissent à la concentration de la production sur le littoral.

Le Japon, qui importe totalement ses matières premières, installe de vastes combinats dans les baies de la façade Pacifique, le plus souvent en gagnant les terrains sur la mer par remblaiement ${ }^{12}$. Organisée autour des trois grands pôles de Tokyo, Nagoya et Osaka, la mégalopole dans l'espace japonais s'en trouve renforcée.

En Europe, les implantations littorales bouleversent le tissu industriel minier et sidérurgique hérité de la première révolution industrielle. Elles sonnent la reconversion des bassins miniers intérieurs dans le Nord de la France, en Lorraine ou dans la Ruhr dont l'exploitation n'est plus rentable par comparaison avec l'importation de charbons ou de minerais en provenance de pays lointains (Afrique du Sud, Colombie, Australie...) mais qui bénéficient de gisements massifs à ciel ouvert.

L'importation de pétrole brut en provenance des pays producteurs aboutit à la concentration des filières pétrochimiques dans les ports avec des prolongements le long des corridors fluviaux, notamment en Europe où sont localisées les plus grandes villes à la différence des Nouveaux Mondes où ces dernières sont majoritairement littorales.

Les ZIP terminent en quelque sorte la première révolution industrielle. Les sites intérieurs de production sont rationalisés et massifiés afin de profiter des économies d'échelle par leur délocalisation sur le littoral qui n'est rendue possible que par des acheminements maritimes à très bas coûts. La géographie portuaire s'en trouve aussi modifiée. Seuls les ports qui bénéficient de vastes espaces bénéficient de la création des ZIP et de la concentration des investissements industriels.

La seconde période débute avec les deux chocs pétroliers. Les pays producteurs de pétrole et les pays émergents se dotent de leurs propres capacités sidérurgiques et de raffinage en suivant le modèle maritime de la ZIP. À l'inverse, en Europe, aux États-Unis ou au Japon, les moteurs de la croissance ne sont plus basés sur les industries lourdes avec comme conséquences une diminution des besoins en acier, un accroissement de l'efficacité énergétique et une modification du mix énergétique pour s'affranchir en partie de la dépendance pétrolière (nucléaire en France ou pétrole de schiste aux États-Unis par exemple) et plus récemment pour répondre à la transition énergétique.

Les pays européens sont en première ligne. Les marchés intérieurs y sont trop limités. Les grands groupes pétroliers ou sidérurgiques raisonnent à l'échelle mondiale surtout lorsque leurs capitaux ne dépendent pas de la localisation de leurs implantations industrielles. Par exemple les groupes Arcelor Mittal ou Tata Steel dominent désormais la sidérurgie européenne. Les crises de surcapacité, alimentées notamment par la Chine, prennent une dimension mondiale par la capacité généralisée du transport maritime à décloisonner les marchés. Elles aboutissent à la rationalisation

\footnotetext{
${ }^{11} \mathrm{~J}$. Malézieux, «L'industrialisation portuaire et les nouvelles relations à la mer : le cas des zones industrialo-portuaires de front de mer », Anthropologie maritime, n², 1985, p.79-85.

${ }^{12}$ P. Pelletier, « De l'Asie des moussons à l'Asie orientale », in P. Pelletier (dir.), Identités territoriales en Asie orientale, Paris, Les Indes savantes, 2004, p.351-372.
} 
des appareils de production par la fermeture des sites les plus fragiles et par la concentration des investissements sur les sites les plus importants. Pour la sidérurgie, cela se traduit par l'extinction des hauts-fourneaux intérieurs comme ceux de Florange ou de Liège dès 2011 et pour le raffinage par la fermeture des plus petites unités de production comme la raffinerie Total de Dunkerque en 2010 ou celle de Pétroplus en 2012 à Rouen.

Dans les dix prochaines années, la poursuite des tendances lourdes actuelles pourrait aboutir à la fermeture d'unités de grande taille situées dans les ZIP européennes si les surcapacités mondiales persistent. Sont en jeu pour les pays concernés des questions d'indépendance nationale et d'affaiblissement de leur tissu industriel et portuaire. À ce titre, le cluster pétrochimique d'Anvers-Rotterdam qui concentre $30 \%$ des capacités européennes de raffinage et où se localise le marché Spot du pétrole pour l'Europe, est mieux placé pour résister que les sites portuaires nationaux plus dispersés des autres États européens.

\subsection{La conteneurisation : mondialisation de l'économie et de l'espace maritime}

Avant l'avènement de la conteneurisation, pendant les années 1950, l'espace maritime est centré sur l'Atlantique Nord qui relie les deux plus gros foyers économiques mondiaux. Les deux autres segments Est-Ouest, transpacifique et Europe/Extrême-Orient, n'ont pas la puissance de l'axe nord-atlantique même si le Japon renaît rapidement de ses cendres. Ailleurs, les liens maritimes restent marqués par le pacte colonial et des liens exclusifs entre les métropoles britannique, française ou néerlandaise et leurs empires respectifs. Les marchés maritimes, reflets de l'organisation commerciale, sont cloisonnés. Assez comparable à celui qui existait avant-guerre, l'espace maritime mondial des années 1950 est caractérisé par la segmentation des différentes routes maritimes qui constituent autant de marchés différents ${ }^{13}$.

À partir des années 1960, l'espace maritime s'internationalise. La croissance du commerce international, la disparition des empires coloniaux, l'émergence des NPIA jouent un rôle évident dans cette internationalisation. Mais la conteneurisation en est aussi un puissant facteur ${ }^{14}$. Elle nécessite des moyens techniques et financiers conséquents et des volumes de marchandises importants à transporter. Les armements s'allient au-delà de leur appartenance nationale dans des consortiums internationaux pour mettre leurs moyens en commun et desservir de vastes marchés, de façade maritime à façade maritime. La fin des paquebots sur l'Atlantique Nord et l'affirmation du Japon comme seconde puissance économique mondiale tendent à atténuer la prépondérance de la route nord-atlantique au profit des deux autres segments Est-Ouest. L'économie mondiale s'organise progressivement autour des trois grands pôles de l'Amérique du Nord, de l'Europe et du Japon mais les trois principales routes maritimes fonctionnent encore indépendamment l'une de l'autre.

Depuis les années 1980 et jusqu'à aujourd'hui, l'Asie orientale est au cœur du système conteneurisé mondial. Les NPIA puis la Chine se sont imposés sur la scène du commerce international tout en tirant la croissance mondiale. De nombreuses zones manufacturières sont situées à proximité immédiate des terminaux à conteneurs afin de faciliter l'exportation des marchandises, notamment dans les zones économiques exclusives chinoises où a démarré l'ouverture de l'empire du Milieu sur le monde à partir de 1978.

Chacun de ces pays est doté d'une ou deux compagnies maritimes de conteneurs considérées par les gouvernements comme

\footnotetext{
${ }^{13}$ A. Frémont, La French Line face à la mondialisation de l'espace maritime, op. cit.

${ }^{14}$ M. Levinson, THE BOX. Comment le conteneur a changé le monde, Paris, Max Milo, 2011.
} 
stratégiques pour l'acheminement du commerce extérieur d'économies encore très extraverties. La construction navale participe de cette stratégie pour s'imposer sur la scène mondiale et remettre en cause l'hégémonie occidentale. Le Japon, la Corée du Sud et la Chine montent successivement en puissance sur ce marché, y sont tout à la fois concurrents et complémentaires, pour contrôler aujourd'hui $90 \%$ du carnet mondial de commandes des pétroliers, vraquiers et porte-conteneurs ${ }^{15}$.

Les armements asiatiques deviennent dominants. Ils s'imposent sur le marché en cassant les taux de fret (les prix) grâce aux surcapacités permises par les constructions de porte-conteneurs. Ils mettent de fait un terme aux vieilles conférences maritimes ${ }^{16}$. Ces quasi-cartels, hérités du XIX ${ }^{\mathrm{e}}$ siècle, permettaient jusqu'alors de fixer les prix par des discussions fermées entre un club limité de compagnies maritimes centenaires, le plus souvent européennes, qui se pensaient éternelles et n'avaient en rien anticipé ce tsunami asiatique.

La géographie des routes maritimes s'en trouve modifiée. À partir de l'Asie orientale partent les deux plus grandes routes conteneurisées transocéaniques, la route transpacifique et celle entre l'Asie orientale et l'Europe. La route de l'Atlantique Nord complète l'artère circumterrestre de circulation des marchandises qui relie les trois pôles majeurs de l'économie mondiale. Mais elle devient progressivement et jusqu'à aujourd'hui presque secondaire. Les liaisons Nord-Sud et Sud-Sud ne comptent que pour $20 \%$ du trafic total. Mais elles ne cessent d'augmenter en valeur absolue dans le contexte généralisé de croissance des trafics conteneurisés. Les déséquilibres de trafic sur chacune de ces routes ne font que refléter ceux du commerce international.

L'espace maritime se mondialise aussi sous l'effet des stratégies des armements, opérateurs de conteneurs. Ils assurent une présence simultanée sur les trois grandes routes maritimes Est-Ouest notamment en se regroupant comme dans le transport aérien dans des alliances d'une ampleur inédite ${ }^{17}$. À partir des années 1990, trois armements européens Maersk Line, MSC et CMA-CGM prennent une dimension mondiale en maillant leur réseau maritime respectif grâce à la technique des hubs, elle aussi empruntée à l'industrie aérienne ${ }^{18}$. Présents sur les trois grandes routes Est-Ouest, ils le sont aussi sur les marchés Nord-Sud moins concurrentiels et donc plus lucratifs car les taux de fret y sont souvent plus élevés. Ils remettent en cause la domination des armements asiatiques, trop centrés sur leurs marchés nationaux et pas assez mondialisés.

\subsection{Un marché conteneurisé mondialisé et oligopolistique}

Le secteur du transport conteneurisé a atteint un degré de concentration inégalé. Les cinq premières compagnies mondiales détiennent en 2018 près de $64 \%$ de la capacité mondiale de transport conteneurisé, ce qui correspond à plus que la part des 20 premiers en 2005 ! Celle des cinq premiers ne s'élevait qu'à $24,3 \%$ en 2000 et $14 \%$ en 1990 . Le transport maritime conteneurisé est une économie à rendements croissants qui tend naturellement vers la situation de monopole. L'économie d'échelle générée par des navires toujours plus grands et les crises cycliques

\footnotetext{
${ }^{15}$ C. Comtois, P. J. Rimmer, "China's competitive push for global trade. Port system development and the role of COSCO", in D. Pinder and B. Slack (eds), Shipping and Ports in the Twenty-first Century. Globalisation, technological change and the environment, London and New York, Routledge, 2004, p. 40-62.

${ }^{16}$ P. Bauchet, Le transport international : dans l'économie mondiale, Paris, Economica, 2e édition, 1991.

${ }^{17}$ T. D. Heaver, H. Meersman, E. Van De Voorde, "Co-operation and competition in international container transport: strategies for ports", Maritime Policy and Management, vol. 28, n 3, 2001, p. 293-305.

${ }^{18}$ D. K. Fleming, Y. Hayuth, "Spatial Characteristics of Transportation Hubs: Centrality and Intermediacy", Journal of Transport Geography, vol. 2, n 1, 1994, p. 3-18.
} 
Carte 1 : La diffusion de la conteneurisation 1970-2003

Trafic des principaux ports à conteneurs du monde (en milliers d'EVP)

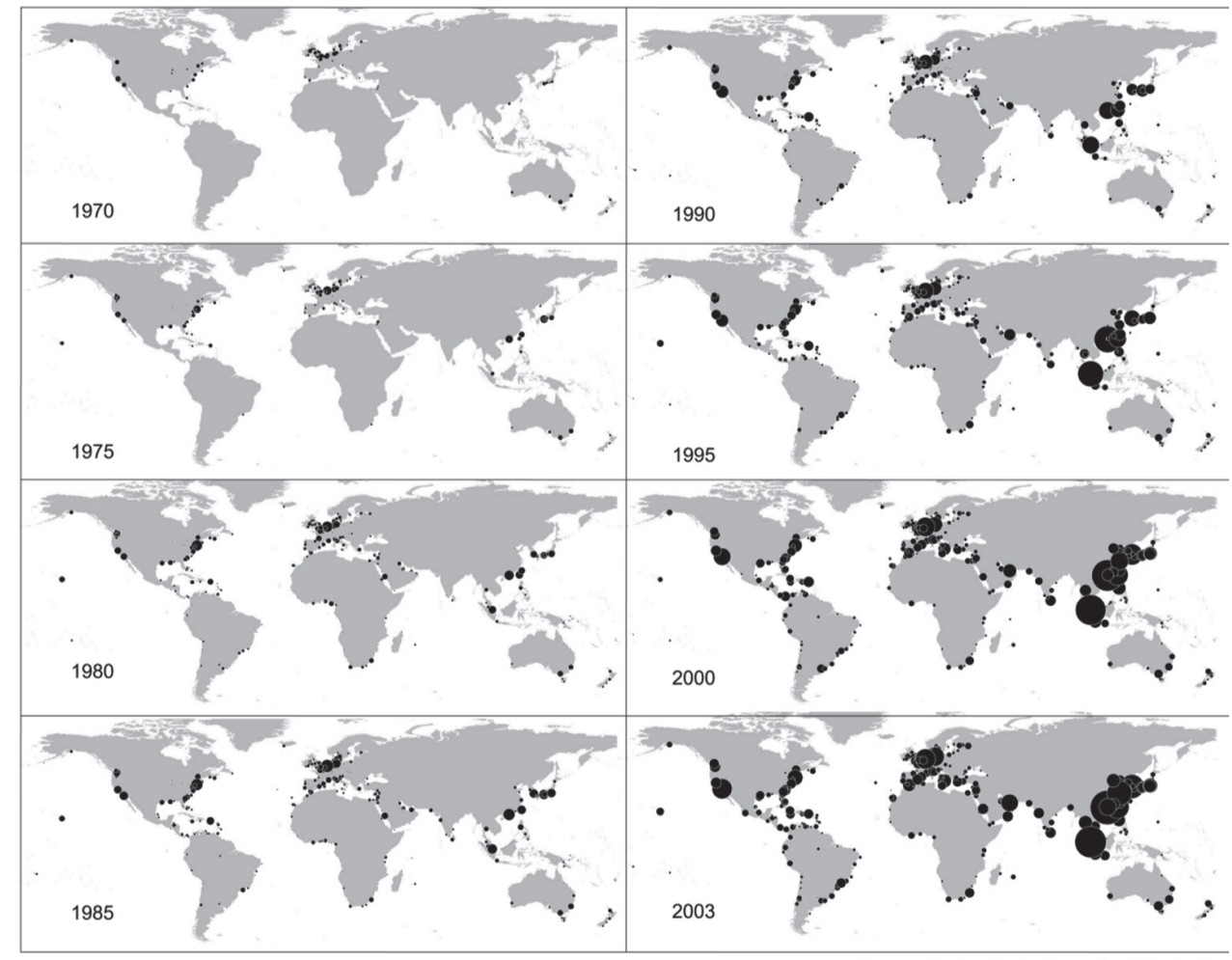

EVP milliers 20449

(2) $\begin{array}{r}1510 \\ 1\end{array}$

Source : A. Frémont, Le monde en boîtes. Conteneurisation et mondialisation, Arcueil, Synthèse INRETS n`53, 2007.

Carte 2 : La circulation des porte-conteneurs le 4 juillet 2012

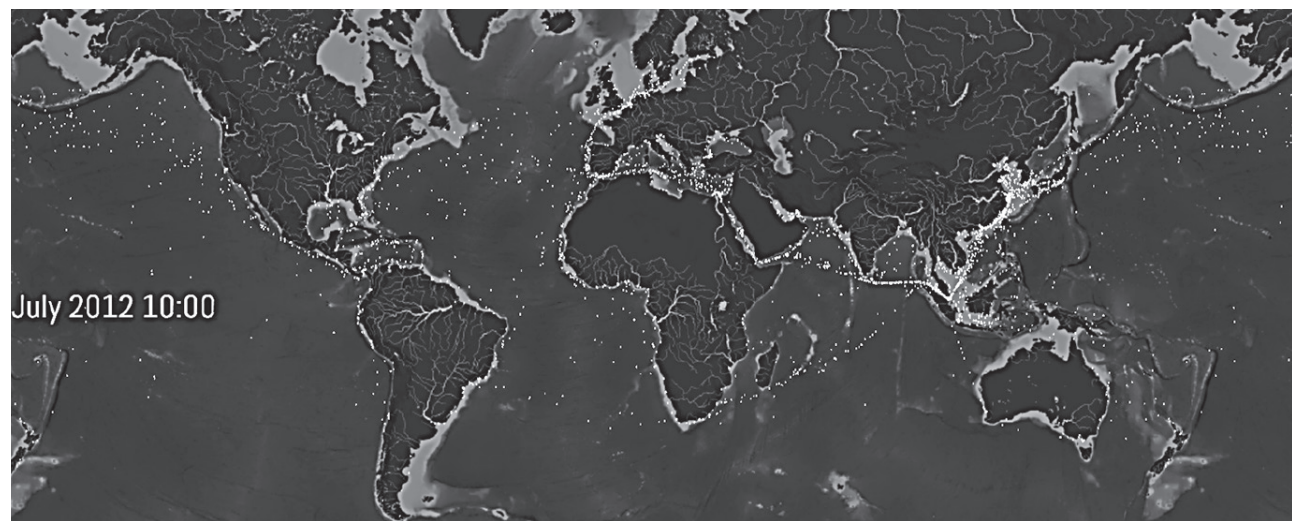

Source : https://www.shipmap.org/ 
de surcapacité débouchent sur des processus récurrents de concentration depuis les années 1970. La crise de 2008 n'a fait qu'accélérer dramatiquement le processus ${ }^{19}$.

Cette concentration du marché est-elle problématique pour les chargeurs ou l'acheminement du commerce extérieur des États ? En 2014, le gouvernement chinois semble avoir répondu positivement à cette question en refusant l'alliance proposée alors par les trois premiers armements mondiaux (Maersk, MSC et CMA-CGM) alors qu'à l'inverse, les autorités de régulation américaines et européennes avaient donné leur accord. La Chine ne souhaitait sans doute pas dépendre de trois opérateurs privés, familiaux et européens.

Les très grands porte-conteneurs nécessitent aussi une modernisation et une adaptation constantes des installations portuaires. Or la concurrence portuaire est la règle et les ports sont dans la dépendance des armateurs ${ }^{20}$. Si les armateurs réalisent des économies d'échelle sur mer, ils reportent de fait sur la partie portuaire et terrestre des coûts logistiques qu'ils ne supportent pas mais qui le sont pour beaucoup par des deniers publics, au nom, à juste titre, de la compétitivité économique et de l'intérêt stratégique du commerce extérieur, de l'emploi et de l'indépendance nationale. La dépendance par rapport à ces grandes compagnies maritimes et la concurrence anesthésient toute esquisse de réflexion collective en matière d'aménagement portuaire qui pourrait se conduire par exemple à l'échelle de l'Union européenne.

\subsection{Les questions sociales et environnementales}

Depuis bien plus longtemps que les GAFA d'aujourd'hui, le transport maritime tire parti des inégalités du monde, qu'elles soient politiques, fiscales, économiques ou sociales, en échappant aux régulations nationales. Secteur international, il « localise » ses activités dans les pays les plus offrant fiscalement et socialement grâce aux pavillons de libre immatriculation. En 2016 et en tonnes de port en lourd, $42 \%$ de la flotte mondiale est sous pavillon du Panama, du Libéria et des Iles Marshall. Plus de $90 \%$ des navires contrôlés par des intérêts localisés dans des pays comme les États-Unis, l'Allemagne ou le Japon sont immatriculés sous des pavillons étrangers ${ }^{21}$. Ces pavillons permettent aux armements d'employer des marins du Tiers-Monde, philippins pour beaucoup. Force est de reconnaître que ces derniers y trouvent des salaires intéressants au prix d'un travail très intense.

Les grandes compagnies maritimes n'ont pas intérêt à exploiter des navires poubelles. En effet, elles redoutent des accidents et les pollutions associées qui ternissent leur image profondément. Mais pour en arriver à cette prise de conscience, quelques catastrophes maritimes retentissantes à proximité des littoraux des pays riches (Exxon Valdez en 1989, Erika en 1999) ont été nécessaires. Les ÉtatsUnis et l'Union européenne ont érigé leurs propres réglementations pour les navires qui fréquentent leurs eaux territoriales. Dans le cadre multilatéral de l'Organisation Maritime International (OMI), il a fallu des années pour mettre au point en 1973 la convention MARPOL, ratifiée près de dix années plus tard. Depuis, cette convention n'a cessé d'être renforcée, notamment en matière de sécurité et de compétence des équipages. Force est là aussi de reconnaître que, d'après l'ITOPF (The International Tanker Owners Pollution Federation) qui compile depuis 1970 les rejets d'hydrocarbures en mer à la suite de l'accident en 1968 du Torrey Canyon, ces rejets ont atteint aujourd'hui un niveau historiquement

\footnotetext{
${ }^{19}$ S. H. Shin, P.T.W. Lee, S. W. Lee "Lessons from bankruptcy of Hanjin Shipping Company in chartering”, Maritime Policy \& Management, vol. 46, n², 2019, p. 136-155.

${ }^{20}$ B. Slack, "Pawns in the Game: Ports in a Global Transportation System", Growth and Change, vol. 24, n 4, 1993, p. 579-588.

${ }^{21}$ Institute of Shipping Economics and Logistic, Shipping Statistics Yearbook 2016, Bremen.
} 
bas même s'il reste difficile d'évaluer les rejets illicites en petites quantités aux conséquences catastrophiques pour le milieu marin.

Aujourd'hui, l'attention se focalise sur les émissions des navires. Ils utilisent des fiouls lourds peu raffinés, peu chers mais fortement émetteurs de soufre. Dans les ports, ils constituent des sources majeures de pollution locale de l'air. « À Marseille par exemple, les émissions de polluants atmosphériques liées au transport maritime représentent $20 \%$ des émissions d'oxydes d'azote, $70 \%$ des émissions d'oxydes de soufre, et $\%$ des émissions primaires de particules fines $\left(\mathrm{PM} 10^{22}\right) »^{23}$. Selon France nature Environnement, un bateau de croisière consommant en moyenne 2000 litres par heure en mer et 700 litres à quai, polluerait autant qu'un million de voitures.

À cela s'ajoute la problématique du réchauffement climatique. Le transport maritime rejette un milliard de tonnes dioxyde de carbone $\left(\mathrm{CO}_{2)}\right.$ par an dans l'atmosphère, soit $2,5 \%$ des émissions mondiales, ou plus que l'Allemagne qui occupe la sixième place dans le classement mondial des pays émetteurs de $\mathrm{CO}_{2} \cdot{ }^{24}$.

L'annexe VI de la convention MARPOL, fixe les limites pour les émissions d'oxydes de soufre (SOx) et d'oxydes d'azote (NOx) provenant des gaz d'échappement des navires. À son entrée en vigueur en 2005, la teneur en soufre maximale des combustibles pour les navires ne doit pas dépasser $4,5 \%$ en masse (45 000 parties par million ou ppm). En 2012, cette teneur a été ramenée à $3,5 \%$ et ne devra pas dépasser $0,5 \%$ en 2020 . Depuis 2015, l'OMI a désigné quatre zones de contrôle des émissions : la mer Baltique, la mer du
Nord et la Manche, l'Amérique du Nord et la mer Caraïbe. La surveillance doit y être plus rigoureuse et la teneur en soufre ne doit pas dépasser 0,1\% ${ }^{25}$. En dehors de l'OMI, les Chinois ont établi trois zones de contrôle des émissions : le delta de la rivière des Perles, le delta du Yangtze et le golfe du Bohai. La teneur en soufre ne doit pas y dépasser pour l'instant les $0,5 \%$. De même, l'OMI encourage le renforcement de l'efficacité énergétique des navires afin de réduire les émissions de $\mathrm{CO}_{2}$. Cette dernière devrait avoir augmentée de $20 \%$ en 2025 par rapport à 2014 .

Pour être efficaces, toutes ces réglementations, qu'elles concernent les navires ou leurs équipages, nécessitent des contrôles. Ceux-ci reposent sur l'État du port (l'État dans lequel se trouve le navire) ou l'État du pavillon. Seuls les États riches disposent de ces moyens de contrôle. Encore hésitent-ils souvent à les employer afin de ne pas faire de tort à leurs ports en concurrence avec ceux des voisins ou même à ne pas les employer du tout pour créer un avantage compétitif. Cela est particulièrement vrai en Europe. La pression des ONG et des opinions publiques, notamment celles des villes portuaires s'avère donc primordial pour le respect des conventions internationales. Dès les années 1990, le port de Los Angeles, totalement cerné par l'urbanisation, a été l'un des premiers à prendre des mesures pour limiter les pollutions des navires afin de mieux pérenniser son activité susceptible d'être remise en cause par les populations. De leur côté, les armateurs ne sont sensibles qu'aux coûts : plus les prix de l'énergie seront élevés, plus ils seront enclins à réduire les consommations de leurs navires par nécessité.

\footnotetext{
${ }^{22}$ PM10 ou Particulate Matter 10 sont des particules en suspension dans l'air dont le diamètre est inférieur à 10 micromètres.

${ }^{23}$ Ministère de la Transition Écologique et Solidaire (MTES), « Restitution de l'étude sur la mise en place d'une zone de réglementation des émissions des navires en Méditerranée », 18 janvier 2019. https://www.ecologique-solidaire. gouv.fr/sites/default/files/DP-ECA\%20MED-180119.pdf.

${ }^{24}$ European Commission, Reducing emissions from the shipping sector, https://ec.europa.eu/clima/policies/transport/ shipping_en\#tab-0-0

${ }^{25}$ OMI, « Nouveau plafond de la teneur en soufre du fuel-oil utilisé par les navires à partir du 1 er janvier 2020 ». http://www.imo.org/fr/MediaCentre/HotTopics/GHG/Pages/default.aspx
} 
Il n'est donc pas étonnant que la face la plus noire du transport maritime concerne d'abord les plus déshérités : pays pauvres desservis par des compagnies maritimes de second rang, utilisant de vieux navires pour des trafics parfois illicites (déchets), marins abandonnés à leur sort en cas de faillite du transporteur, démantèlement des navires en Inde dans des conditions calamiteuses pour les ouvriers et l'environnement. En 2006, l'affaire du Probo Koala avait par exemple révélé comment il était possible de se débarrasser d'une cargaison toxique, résultant d'une opération de raffinage effectuée plusieurs mois auparavant dans les eaux internationales. Les 530 tonnes, déversées de nuit dans des décharges d'Abidjan, avaient provoqué la mort de 17 personnes et l'intoxication de milliers d'autres. Ces déchets auraient dû être traités dans les installations spécialisées d'un port européen.

\section{CONCLUSION}

Depuis la fin de la Seconde Guerre mondiale, le transport maritime a été un facteur puissant de la mondialisation. Il a rendu techniquement possible cette dernière. Il a aussi durablement contribué à la façonner en redessinant l'espace productif mondial. L'industrie maritime s'est elle-même profondément transformée avec un bouleversement des hiérarchies maritimes au profit de l'Asie orientale. L'espace maritime, de segmenté qu'il était dans les années 1950, est désormais un espace lui-même mondialisé.

La conteneurisation a joué un rôle moteur et particulier dans ces transformations. Cette innovation a rendu possible l'internationalisation des chaînes de valeur pour les produits manufacturés et semi-finis qui depuis les années 1970 sont au cœur des économies les plus avancées. Elle s'appuie sur la massification tout en autorisant une collecte ou distribution très fine de ces mêmes produits dans les territoires. Elle va de pair avec le développement de la logistique qui est désormais une composante des systèmes de production et de distribution. Sans transport maritime et sans conteneurisation, la mondialisation n'aurait pas été possible ou elle aurait pris une forme différente.

Secteur international au service du commerce international, très concurrentiel et bénéficiant de la liberté des mers, le transport maritime est difficile à réguler alors même que ses impacts sont considérables et multiples. Préserver cette liberté a un prix finalement relativement faible pour cette industrie qui a tout intérêt à éviter les catastrophes ou plus simplement à ne pas trop attirer l'attention sur elle. Elle tente actuellement de relever le défi environnemental sous la pression des gouvernements, des ONG et des opinions publiques des pays riches qui dans le même temps sont très satisfaits d'un système qui permet de consommer des produits du monde entier. «Pour vivre heureux, vivons cachés ». 